\title{
Stochastic optimization of carbon mitigation path in Shenzhen based on uncertainty of power demand
}

\author{
Qinghui $\mathrm{Hu}^{1}$, Yushen Tian ${ }^{2 *}$ \\ ${ }^{1,2}$ School of Environment and Energy, Peking University, Nanshan District, Shenzhen, China
}

\begin{abstract}
The core elements of urban carbon emission mitigation optimization path include structural adjustment, low energy supply, technological innovation, and enhanced energy demand management and improvement. How to optimize the combination of these factors to achieve the city's emission mitigation goals at the lowest cost is very important to study the path of urban low-carbon development. Due to many factors involved, it is difficult to solve this problem by building a mathematical optimization model that includes all the elements. This paper minimizes the total cost of emission mitigations in various departments in Shenzhen, combines the uncertainty of parameters and constraints, and uses mathematically standardized method to establish a stochastic optimization model for urban carbon emissions paths. Considering the uncertainty of energy demand, the optimal promotion rate of technical measures of the city's various departments in the stochastic optimization model during the planning period can be obtained, and the optimal solution of the city's lowcarbon development optimization path can be formed.
\end{abstract}

\section{Introduction}

With the acceleration of urbanization and industrialization, China's energy demand and consumption have gradually increased, and the contradiction between energy supply and demand has become increasingly intensified. Among all industries in the city, the carbon dioxide emissions of the power, manufacturing, transportation, and construction sectors are relatively large. At the same time, these industries also have large emission mitigation potentials. To achieve the urban energy conservation and emission mitigation goals, the key is to reduce carbon emissions of these industries, but the promotion of emission mitigation technology requires capital investment. While promoting energy conservation and carbon emission mitigation, we need to control emission mitigation costs. Therefore, how to achieve the maximum emission mitigation at the lowest cost is worthy of further study.

Many scholars have studied the carbon emission mitigation path through model. The research of foreign scholars mainly focuses on the power sector. Zhang et al. [1] established a multi-period model and optimization framework for China's power industry. Jin et al. [2] developed Carbon Capture and Storage technology based on carbon mitigation targets and

Corresponding author: tianyushen1211@pku.edu.cn 
minimizing construction costs. Shi et al. [3] proposed energy systems integration considering the uncertainty of energy supply and demand. Cheng et al. [4] considered changing the investment decision to make a development plan for the production period from 2011 to 2050 . Lee [5] presented a multi-period power plant planning based on carbon capture (CC) technologies. Zhang et al. [6] proposed a multi-period optimization model of China's power sector by considering the uncertainties of power demand, fuel prices and carbon tax policy. Some domestic scholars also research the development plan of the power sector. For example, Qiu et al. [7] presented a grid planning model that satisfies the system reliability criteria as the constraint and the construction costs of each stage of the system development process as the optimization goal. At the same time, some scholars turned their research objects to other departments. Ma et al. [8] used the optimization mathematical method and explored the optimal path of carbon emission mitigation in Shenzhen manufacturing. Tang et al. [9] selected 42 sectors to build a input-output model of multiobjective based on maximizing economic development and minimizing carbon emissions as optimization goals.

However, due to the availability of data, there is no article that studies carbon emission mitigation technologies in detail, and related research often focuses on one sector, and lacks overall research on multiple sectors. At present, Shenzhen is forming a win-win situation for environmental protection and economic development. Studying Shenzhen's low-carbon development path lends reference to other cities. Therefore, this paper establishes a stochastic optimization model with the goal of minimizing the total cost of emission mitigation in four sectors of Shenzhen by using the promotion rate of each carbon emission mitigation measure as an optimization variable. Combining the uncertainty of parameters and constraints, mathematical methods are used to determine the theoretical optimal values. After the theoretical optimal values of the promotion rate of emission mitigation measures in four sectors are calculated, the theoretical optimal solution of the emission mitigation path is determined, which can be used as a theoretical basis for urban carbon emission mitigation work and set energy saving and emission mitigation targets.

\section{Materials and Methods}

\subsection{Model Building}

The structure of the optimization model mainly includes three major elements: objective function, decision variables, and constraints. The objective function of the urban carbon emission mitigation optimization model is to minimize the total cost of each department during the planning period, and the decision variable is the promotion rate of each department's technology and other measures. Constraints mainly include carbon emission constraints, fuel supply constraints and non-negative constraints.

For the convenience of description, the physical meaning of each variable in the model is given in Table 1 in the appendix.

The objective function of the urban carbon emission mitigation path optimization model is to minimize the cumulative costs of the power sector, manufacturing sector, transportation sector, and construction sector during the planning period, as shown in equation (1):

$$
C=\sum_{t=2015}^{2030}\left[\frac{\sum_{1}^{n_{1}}\left(t i n v_{t}^{n b}+t i n v_{t}^{\prime f}+t o m_{t}+t f u_{t}+t e x_{t}\right) * x_{i}+\sum_{n_{t}+1}^{n_{2}} \operatorname{tin} v_{t}^{f f} * x_{i}+\sum_{n_{2}+1}^{n_{3}} \operatorname{tin} v_{t}^{\prime f} * x_{i}+\sum_{n_{3}+1}^{n_{4}} t i n v_{t}^{\prime f} * x_{i}}{(1+I)^{t-2015}}\right]
$$

The constraint conditions are shown in the following formula: 
(1) Carbon mitigation constraints. Equations (2) to (5) indicate that the total carbon emission mitigation of each technology in different sectors should be greater than or equal to the planned carbon emissions:

$$
\begin{gathered}
\sum_{t=2015}^{2030} \sum_{i=1}^{n_{1}} \sum_{f} x_{i} * i c_{i} * s a v_{i, f} * e f_{f} \geq F_{t, e}^{p l} \\
\sum_{t=2015}^{2030} \sum_{i=n_{1}+1}^{n_{2}} x_{i} * s a v_{i} \geq F_{t, m}^{p l} \\
\sum_{t=2015}^{2030} \sum_{i=n_{2}+1}^{n_{3}} x_{i} * s a v_{i} \geq F_{t, c}^{p l} \\
\sum_{t=2015}^{2030} \sum_{i=n_{3}+1}^{n_{4}} x_{i} * s a v_{i} \geq F_{t, t}^{p l}
\end{gathered}
$$

(2) Electricity demand constraints. Due to uncertainty of many factors such as the speed of economic growth and future power demand, $\varepsilon_{E D}$ is set to be the stochastic fluctuation of electricity demand, then we can express the power demand constraint more reasonably:

$$
\sum_{k=1}^{m_{1}} i c_{k, t} * O H_{k}+\sum_{k=m_{1}+1}^{m_{2}} x_{k} * I C_{k, t} * O H_{k}+S u_{e x} \geq E D_{t}+\varepsilon_{E D}
$$

For the stochastic fluctuation $\varepsilon_{E D}$, we can use the opportunity constrained programming model to solve it, this model assumes the probability that the constraint is true is greater than or equal to a certain confidence level:

$$
\operatorname{Pr}\left(\sum_{k=1}^{m_{1}} i c_{k, t} * O H_{k}+\sum_{k=m_{1}+1}^{m_{2}} x_{k} * I C_{k, t} * O H_{k}+S u_{e x} \geq P D_{t}+\varepsilon_{P D}\right) \geq \alpha
$$

(3) Installed capacity constraints. Equation (8) indicates that each type of power plant's installed capacity should not exceed its own maximum $I C_{K}^{\max }$ :

$$
x_{k} * I C_{K} \leq I C_{K}^{\max }
$$

(4) Fuel supply restrictions. Equation (9) states that each fuel's supply should not exceed its maximum $F S C \max _{f, t}$ :

$$
\sum_{k=1}^{n} x_{k} * f d_{f, t}^{k} \leq F S C_{f, t}^{\max }
$$

(5) Non-negative constraints. As shown in Equation (10), the promotion rate of various carbon emission mitigation technologies in four sectors should be set between 0 and 1 .

$$
0 \leq x_{i} \leq 100 \%, i=1,2, \ldots
$$

\subsection{Empirical analysis_— taking Shenzhen as an example}

\subsubsection{Carbon mitigation measures}


According to the specific situation of Shenzhen, Carbon mitigation technologies in different sectors are shown in Table 2 in the appendix.

\subsubsection{Data Sources}

First, the list of emission mitigation technologies and indicator for four sectors was collected through field research, enterprise questionnaire survey, expert consultation and literature research. Secondly, other parameters of the power sector were obtained through field surveys from different power plants in Shenzhen. Finally, the planned emission mitigation was calculated according to the outline of the 13th five-year plan for Shenzhen's national economic and social development, the original carbon emission data of Shenzhen and the forecast of Shenzhen's economic growth.

\section{Results and Discussion}

According to the model and data proposed in Section 2, the optimal carbon emission mitigation paths for Shenzhen's power sector, manufacture sector, construction sector and transportation sector under uncertain power demand are obtained.

\subsection{Power Sector}

Figure 1 shows the promotion rate of various emission mitigation technologies in Shenzhen's power sector from 2015 to 2030.

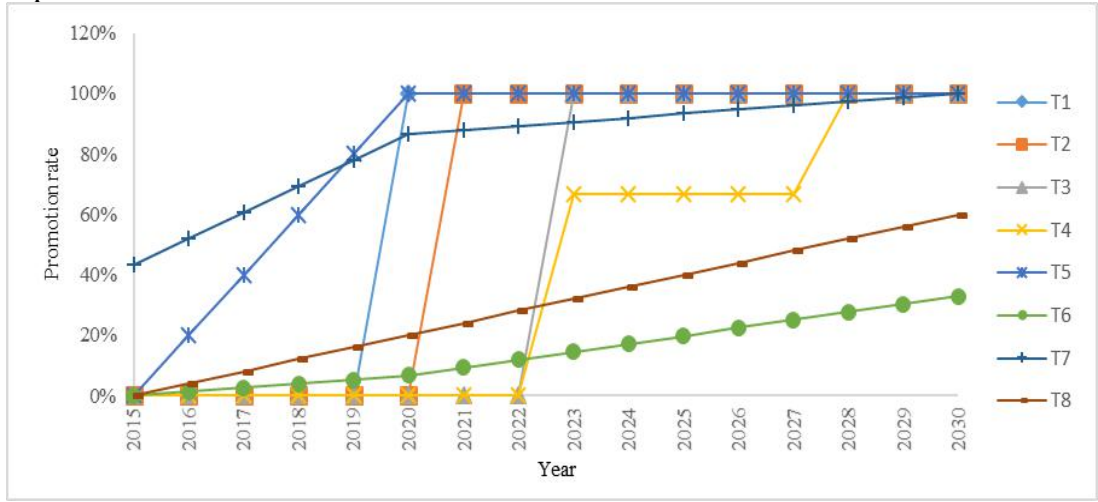

Fig. 1. Promotion rates of different technologies in the power sector

We found that some technologies have the same popularization rate, so different technologies can be combined into 8 subsets, as shown in Table 3. in the appendix.

As shown in Figure 3.1, The promotion rate of technology in subset T1 will first reach $100 \%$ in 2020 , subsets T2 and T3 are implemented immediately after T1. This is because the carbon emission mitigation amount and cost of different technologies are different. The cost of $\mathrm{T} 1$ is relatively low so the carbon mitigation is more efficient. Distributed photovoltaic power generation(T6) is a distributed power generation system that can directly convert solar energy into electrical energy. It is a new type of power generation and comprehensive energy utilization method with broad development prospects. It is limited by the high cost and developed relatively slowly. Waste-to-energy(T7) is a good way to utilize waste. Its development is relatively fast because of benefiting from the government subsidies, which has also accelerated the decommission of the Mawan Power Plant. 


\subsection{Manufacturing, Construction and Transportation sectors}

The promotion rates of various emission mitigation technologies in the manufacturing, construction and transportation sectors from 2015 to 2030 are shown in Figure 2, Figure 3 and Figure 4.

Manufacturing technology is integrated into three subsets, subset T9 includes i31, T10 includes i46, and T11 includes the remaining technologies for manufacturing carbon mitigation. Different technologies in $\mathrm{T} 11$ have the same promotion rate, mainly because their carbon emission mitigation efficiency is relatively low. As shown in Figure 3.2, the technology in subset T10 will be implemented in 2028. Taking into account the discount rate, the amount of investment will decrease over time, which makes the carbon emission mitigation efficiency of T10 continue to improve to be close to T9 in 2028. The technology in $\mathrm{T} 11$ does not improve carbon emission mitigation efficiency over time, so the promotion rate has always been zero.

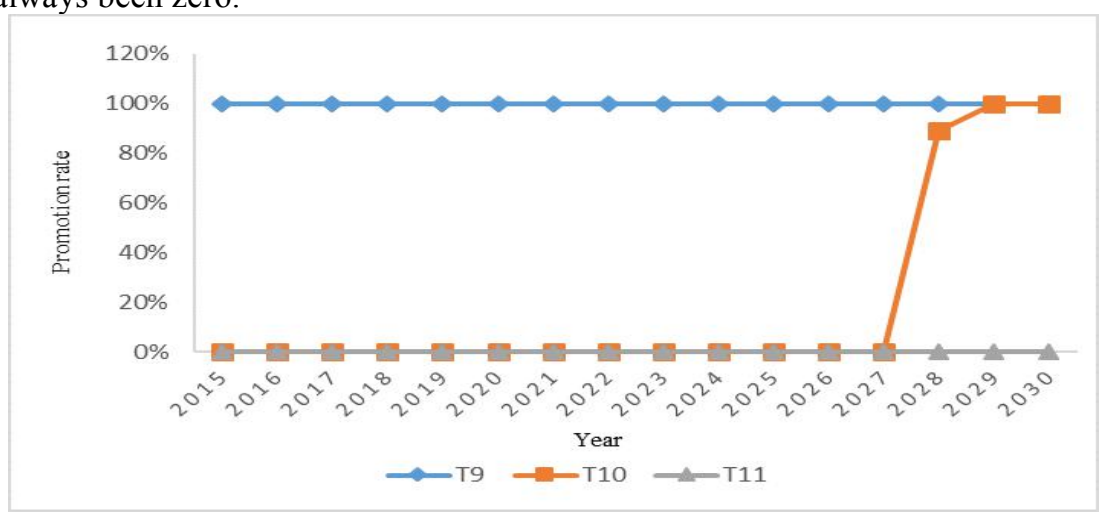

Figure 2. Promotion rates of different technologies in the Manufacturing sector

The technology of the construction sector is integrated into 3 subsets, the subset T12 includes i80, i86, i105, i106, i107, T13 includes i77, and T14 includes the remaining carbon emission mitigation technologies of the construction sector. The promotion rate of T12 technologies during the planning period is $100 \%$, because their investment is relatively low. For example, the cost of the Energy Monitoring Statistics Database is close to zero, so its carbon emission mitigation efficiency is also higher. As shown in Figure 3.3, the technology in the subset T13 will be improved quickly in 2030, and the carbon emission mitigation efficiency of T13 in 2030 will gradually approach that of T12. The technology in T14 does not improve carbon emission mitigation efficiency during the planning period, so the promotion rate has always been zero.

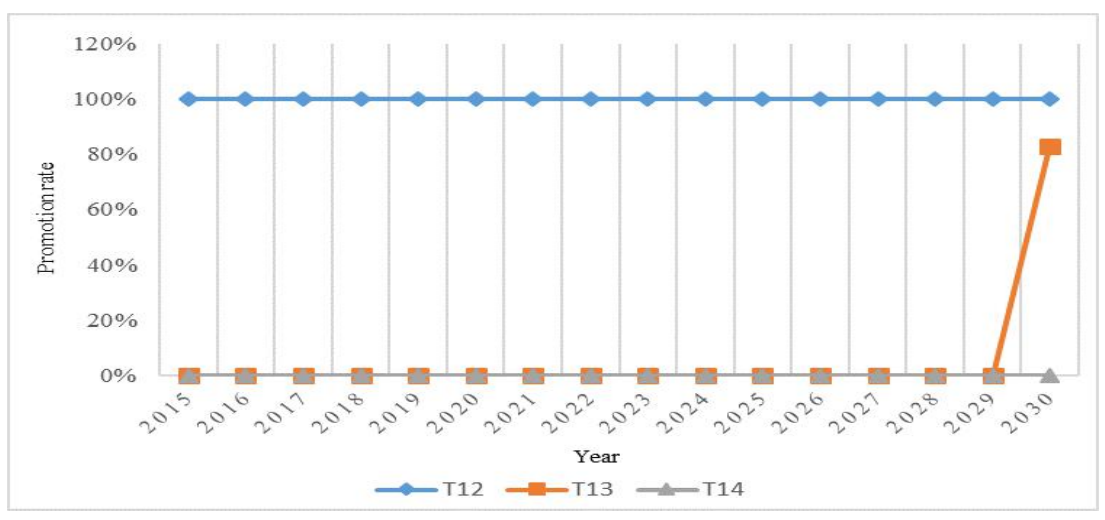


Figure 3. Promotion rates of different technologies in the Construction sector

The technology of the transportation sector is integrated into two subsets. Subset T15 includes i120, i124, i129, i130, i131 and i134-i141, and T16 includes the remaining emission mitigation technologies of the transportation sector. The promotion rate of T15 technologies during the planning period is $100 \%$, because their cost is zero, which also makes carbon emission mitigation efficiency very high. Reducing carbon emissions by managing energy conservation and other zero-cost methods will be an important means of reducing carbon emissions in the transport sector.

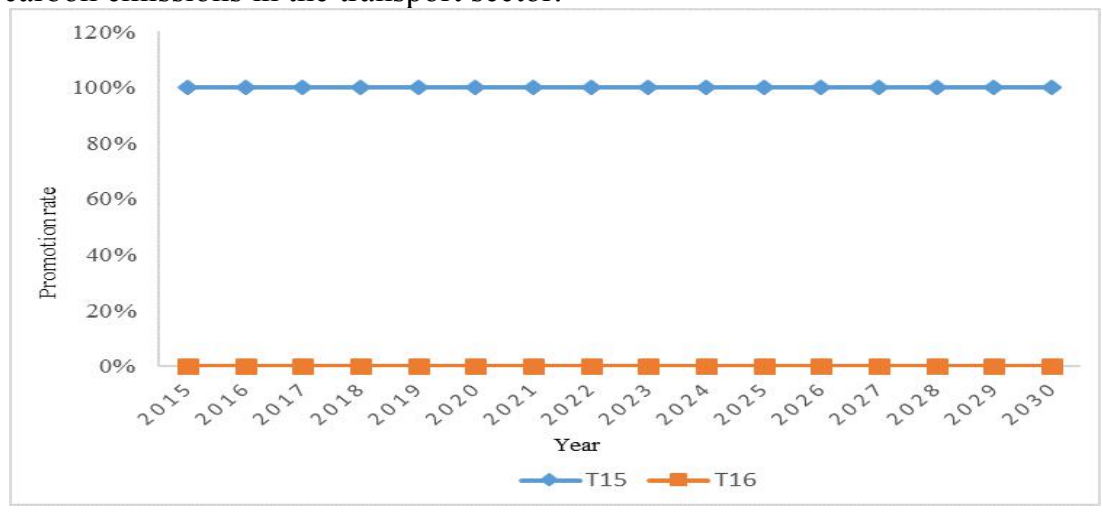

Figure 4. Promotion rates of different technologies in the Transportation sector

\section{Conclusions}

In this paper, considering the uncertainty of power demand and the background of the low-carbon development plan of Shenzhen, a mathematical planning method is used to establish the stochastic optimization model of urban carbon emission paths. We calculate the promotion rate of each emission mitigation measure during the planning period with the goal of minimizing the total cost of reducing emissions in four sectors. Then it can determine the theoretically optimal solution for each emission mitigation subdivision path, which will lay a foundation for the design and evaluation of low-carbon development routes. It also points out the direction for the actual setting of energy-saving emission mitigation targets and the implementation of energy-saving emission mitigation measures.

The empirical analysis of Shenzhen shows that the best promotion strategy for urban emission mitigation measures during the planning period is to promote the emission mitigation measures with the lowest emission mitigation cost and the highest emission mitigation efficiency, and to make their promotion rate reach $100 \%$ as soon as possible. For example, many of the measures preferentially promoted in the transport sector are low-cost policy technologies, on the contrary, the promotion rate of emission mitigation measures with high emission mitigation costs is low, such as T16 emission mitigation measures in the transportation sector. According to this stochastic optimization model, we can get the optimal promotion rate of the technical measures of four sectors in the city during the planning period, and form the optimal solution of the low-carbon development path under certain exogenous conditions. The stochastic model guarantees the robustness of the results and the stability of policy recommendations.

If we promote various energy saving and emission mitigation technologies in accordance with the promotion rate calculated from the optimization model, the planned emission mitigation goals will be completed at the lowest cost during the planning period. However, since the promotion of emission mitigation measures is affected by policies, 
promotion costs and other factors, the promotion of various measures is often different from the ideal situation. In the actual promotion, the city's specific conditions and the efficiency of energy-saving and emission mitigation measures should be fine-tuned to design an optimal path of carbon emission mitigation in line with the city's actual conditions.

\section{Acknowledgements}

This work did not receive any specific grant from funding agencies in the public, commercial, or not-for-profit sectors.

\section{References}

1. D. Zhang, P. Liu, L. Ma, Z. Li, W. Ni, COMPUT CHEM ENG,37(2012)

2. Jin, L. Bai, J. Kim, S. Jeong, K. Kim, ENERGIES,10(2017)

3. J. Shi, Y. Wang, R. Fu, J. Zhang, SUSTAINABILITY-BASEL,9(2017)

4. R. Cheng, Z. Xu, P. Liu, Z. Wang, Z. Li, I. Jones, APPL ENERG,137(2015)

5. J.Y. Lee, APPL ENERG,198(2017)

6. D. Zhang, P. Liu, L. Ma, Z. Li, COMPUT CHEM ENG,50(2013)

7. W. Qiu, Y. Qiu, Zhejiang Electric Power,29(2010)

8. X. Ma, B. Hu, H. S, J. J, Modern Management Science, 11(2016)

9. J. T, B. Z, S. C, Journal of Industrial Technological Economics, 12(2012) 